\title{
Osteomielitis de tibia secundaria a la vacuna BCG en un paciente pediátrico inmunocompetente. Reporte de un caso
}

\author{
Javier E. Dal Lago, Eduardo J. Levy \\ Equipo de Ortopedia y Traumatología Infantil, Hospital Británico de Buenos Aires, Ciudad Autónoma de Buenos Aires, Argentina
}

\begin{abstract}
RESUMEN
Introducción: La vacuna BCG (bacilo de Calmette-Guérin) para prevenir las formas graves de tuberculosis, es la vacuna más difundida en el mundo. Los efectos adversos asociados a la vacunación son poco frecuentes, y la mayoría de ellos ocurren en el sitio de inoculación. Presentamos un caso de osteomielitis de tibia secundaria a la vacuna BCG en un paciente pediátrico inmunocompetente. Conclusiones: El compromiso óseo secundario a la vacuna BCG en pacientes previamente sanos es muy raro. Es importante sospecharlo, para diagnosticarlo y administrar el tratamiento adecuado. Se obtuvieron buenos resultados administrando fármacos antituberculosos, sin necesidad de limpieza quirúrgica.
\end{abstract}

Palabras clave: Tuberculosis; bacilo de Calmette-Guérin; BCG; osteomielitis; osteítis.

Nivel de Evidencia: IV

Tibia osteomyelitis secondary to BCG vaccination in an immunocompetent infant. Case report

\section{ABSTRACT}

Introduction: The Bacillus Calmette-Guérin (BCG) vaccine, used to prevent severe forms of tuberculosis (TB), is the most extensively used vaccine worldwide. Adverse events associated with BCG vaccination are rare, and most of them occur at the inoculation site. We present a tibia Osteomyelitis case secondary to BCG vaccination in an immunocompetent infant. Conclusions: Bone involvement secondary to $B C G$ vaccination in previously healthy patients is extremely rare. Healthcare providers must consider such settings in order to make the diagnosis and institute the appropriate treatment. Antituberculous drugs produced good therapeutic results with no need for surgical toilette.

Key words: Tuberculosis; TB; Bacillus Calmette-Guérin; BCG; osteomyelitis; osteitis.

Level of Evidence: IV

\section{INTRODUCCIÓN}

La tuberculosis es una enfermedad infectocontagiosa, granulomatosa crónica. El agente etiológico es Mycobacterium tuberculosis o bacilo de Koch. La Organización Mundial de la Salud estima que hay 9 millones de enfermos y que se producen 1 millón y medio de muertes por tuberculosis en el mundo, cada año; esto la convierte en una de las enfermedades infecciosas más letales. ${ }^{1}$

Entre las estrategias para disminuir la mortalidad y las formas graves de la enfermedad se cuenta con la vacuna BCG (bacilo de Calmette-Guérin), que contiene microorganismos vivos y atenuados obtenidos a partir de Mycobacterium bovis. En nuestro país, su aplicación es obligatoria en los recién nacidos antes del alta de la maternidad, con el fin de prevenir la bacteriemia por la primoinfección natural por el bacilo de Koch. ${ }^{2}$

A pesar de que la vacuna BCG se usa ampliamente en el mundo y cerca de 100 millones de recién nacidos son vacunados cada año, el porcentaje de efectos adversos asociados es proporcionalmente bajo (Tabla). ${ }^{3}$

Se presenta un caso clínico de osteítis/osteomielitis secundaria a la vacuna BCG en un paciente pediátrico.

Recibido el 17-2-2019. Aceptado luego de la evaluación el 5-5-2019 • Dr. JAVIER E. DAL LAGO • javierdallago @yahoo.com.ar

Cómo citar este artículo: Dal Lago JE, Levy EJ. Osteomielitis de tibia secundaria a la vacuna BCG en un paciente pediátrico inmunocompetente. Reporte de un caso. Rev Asoc Argent Ortop Traumatol 2020;85(2): 157-166. https://doi.org/10.15417/issn. 1852-7434.2020.85.2.965 
Tabla. Efectos adversos de la vacuna BCG

\begin{tabular}{|l|l|l|}
\hline $\begin{array}{l}\text { Naturaleza de los } \\
\text { efectos adversos }\end{array}$ & Descripción & Tasa/dosis \\
\hline Leves & $\begin{array}{l}\text { Pápula en el lugar de la inyección (inicio 2-4 semanas) } \\
\text { Ulceración moderada } \\
(1-2 \text { meses) } \\
\text { Cicatriz (2-5 meses) }\end{array}$ & Casi todos los vacunados \\
\hline Severos & $\begin{array}{l}\text { Locales } \\
\text { Absceso localizado } \\
\text { Cicatriz queloide } \\
\text { Linfadenitis } \\
\text { Supuración (inicio 2-6 meses) }\end{array}$ & \\
\hline & $\begin{array}{l}\text { Sistémicos por 1000-10.000 } \\
\text { (inicio 1-12 meses) } \\
\text { Lesiones cutáneas } \\
\text { Osteítis } \\
\text { BCG generalizada } \\
\text { Síndrome de reconstitución inmune }\end{array}$ & \\
\hline & $\begin{array}{l}\text { Reportes de casos únicamente } \\
1 \text { por 3333-1.000.000 } \\
1 \text { por 230.00-640.000 } \\
1 \text { por 640.000 }\end{array}$ \\
\hline Datos obtenidos de la Hoja de Información sobre las tasas observadas de reacciones a vacunas. Vacuna Bacilo Calmette-Guérin (BCG). OMS 2012.
\end{tabular}

Datos obtenidos de la Hoja de Información sobre las tasas observadas de reacciones a vacunas. Vacuna Bacilo Calmette-Guérin (BCG). OMS 2012.

\section{CASO CLÍNICO}

En abril de 2015, una niña de 9 meses de edad, previamente sana, y con su calendario de vacunación completo fue traída por su madre a los consultorios externos del Servicio de Ortopedia y Traumatología. La madre refirió que, desde hacía unos días, la niña mostraba signos de defensa y dolor a la palpación del tobillo izquierdo. Advertía que ya no intentaba pararse como en días anteriores y arrastraba el miembro inferior izquierdo al gatear. Negaba antecedentes de traumatismos y fiebre.

Clínicamente la paciente mostraba facie de dolor y llanto a la palpación del tercio distal de la pierna. Sin dolor a la movilidad pasiva del tobillo. No se observaron hematomas, edema, ni signos de flogosis local.

Se solicitaron radiografías de ambos miembros inferiores, en las cuales se observó una lesión osteolítica que comprometía la metáfisis distal de la tibia izquierda (Figura 1).

Dado el tipo de lesión revelada por las radiografías, se plantearon como posibles diagnósticos diferenciales tanto cuadros neoplásicos como una infección ósea. Por lo tanto, se decidió internar a la niña para continuar la evaluación con estudios complementarios.

Se solicitaron análisis de laboratorio que arrojaron los siguientes resultados: hematocrito 33\%; hemoglobina $11,5 \mathrm{~g} / \mathrm{dl}$; glóbulos blancos $9000 / \mathrm{mm}^{3}$, plaquetas $565.000 / \mathrm{mm}^{3}$, eritrosedimentación $34 \mathrm{~mm} / \mathrm{h}$ y proteína C reactiva $<0,5 \mathrm{mg} / \mathrm{l}$.

En la resonancia magnética de tobillo izquierdo bajo anestesia general, se observó una lesión bien definida, de $10 \times 8 \mathrm{~mm}$, a nivel diafiso-metafisario de la tibia distal izquierda, que comprometía cartílago de crecimiento con reacción esclerótica y edema medular óseo adyacente (Figura 2).

Mientras la paciente estuvo internada, se inmovilizó su miembro inferior izquierdo con una valva corta de yeso, y se programó una biopsia ósea de la lesión. El análisis directo de la muestra no halló bacterias y la tinción de Ziehl-Neelsen fue negativa para bacterias acidorresistentes.

El estudio anatomopatológico informó una reacción granulomatosa, asociada a granulomas eosinófilos. Estos resultados continuaban dejando como diagnósticos diferenciales a la histiocitosis por células de Langerhans o la osteomielitis granulomatosa.

Después de la biopsia, la paciente evolucionó favorablemente con disminución del dolor local. Se mantuvo una conducta expectante, mientras se continuaban realizando estudios a la espera del cultivo bacteriológico de la muestra obtenida en la biopsia. 


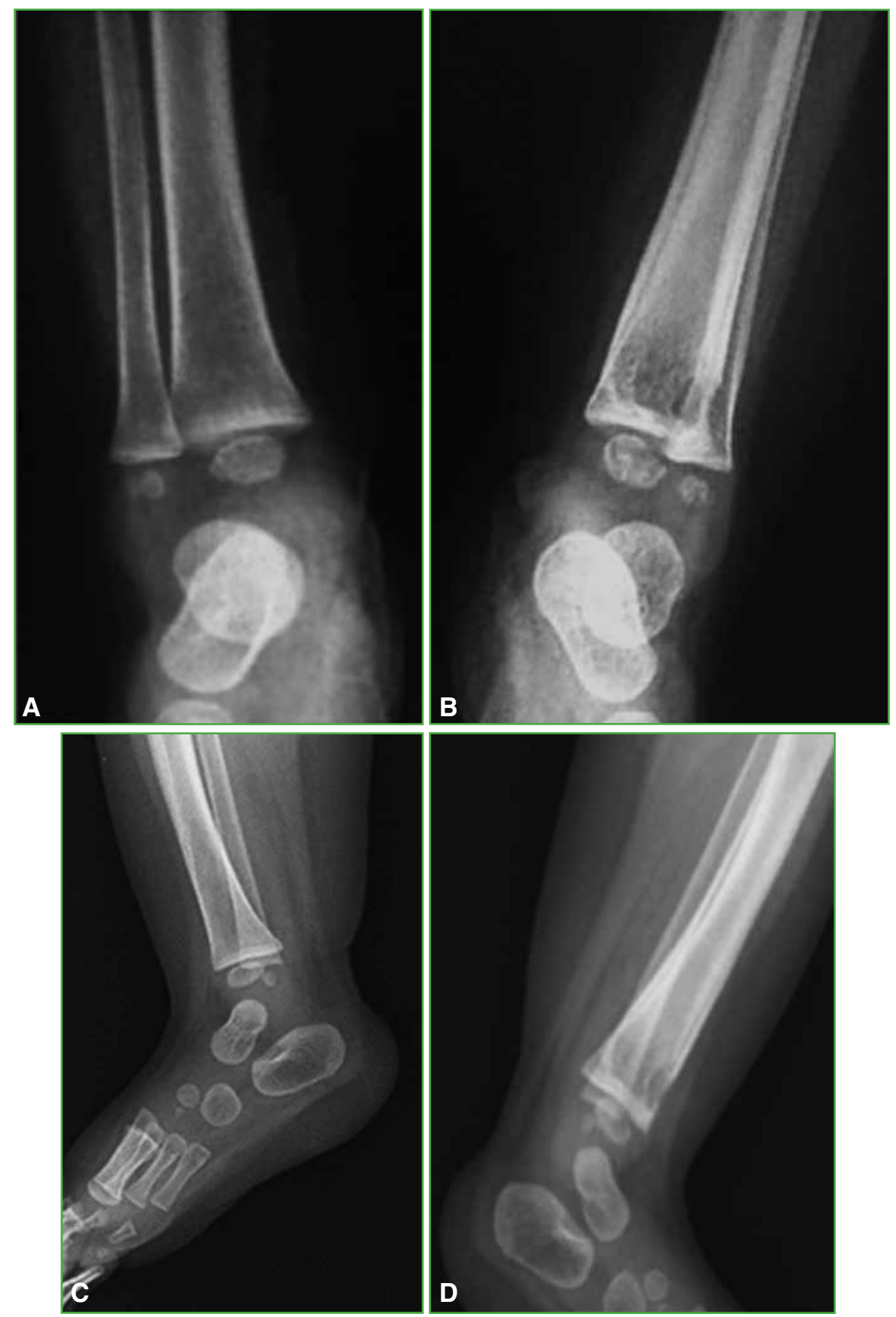

Figura 1. Radiografías de frente y de perfil, de ambos tobillos.

B y D. Se puede observar la lesión osteolítica en la metáfisis distal de la tibia izquierda. 


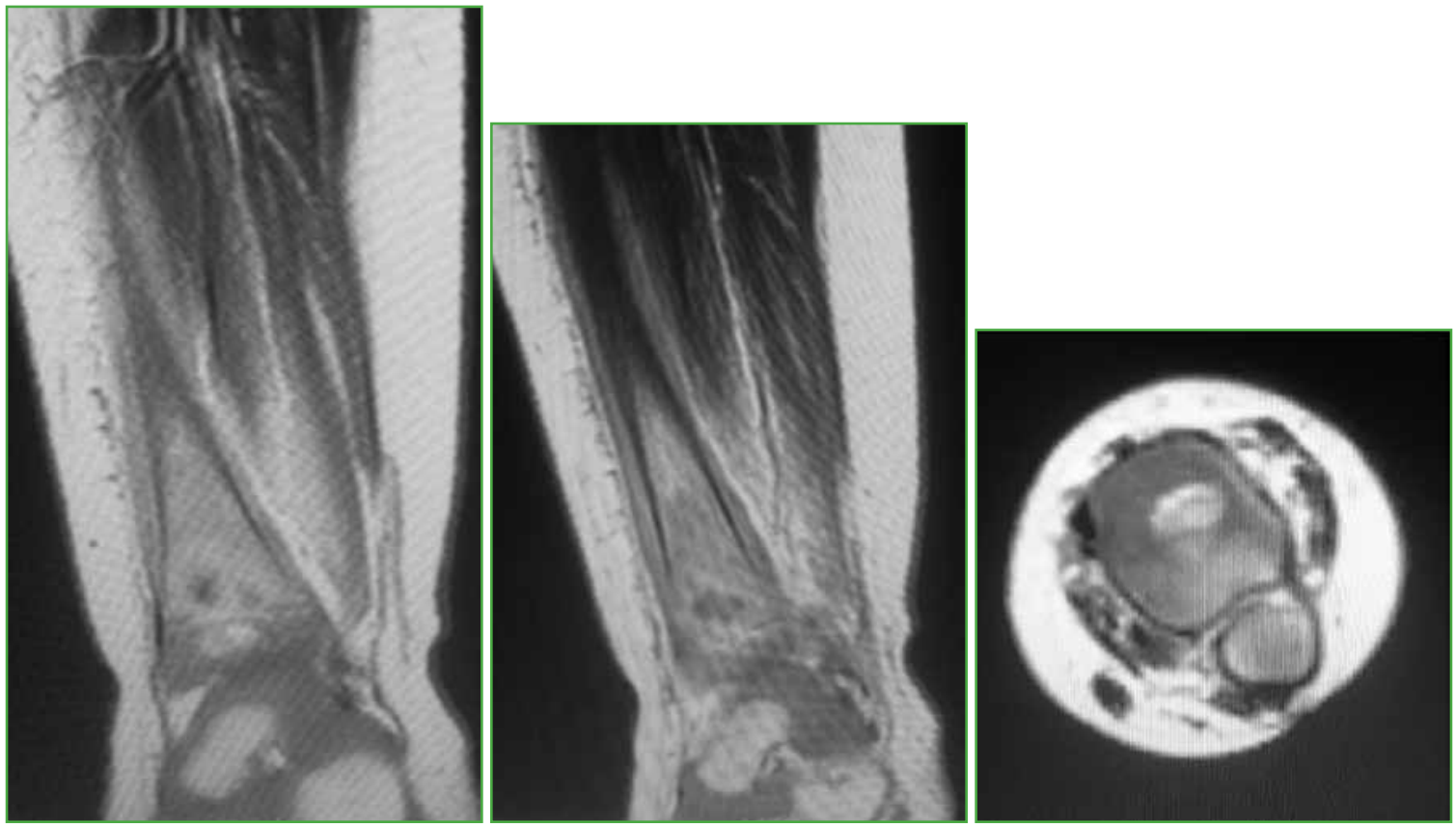

Figura 2. Resonancia magnética de tobillo izquierdo, cortes sagitales y axial.

Se descartó cualquier tipo de inmunodeficiencia. Se solicitaron radiografías de cráneo y de huesos largos, en las que no se detectaron otras lesiones óseas. Los hemocultivos y el cultivo de líquido cefalorraquídeo fueron negativos. El fondo de ojo, la evaluación de los potenciales evocados auditivos y visuales, como así también la radiografía de tórax arrojaron resultados normales.

Luego de tres semanas de incubación, se desarrolló una micobacteria en el cultivo del material obtenido en la biopsia. El estudio genético mediante la reacción en cadena de la polimerasa informó Mycobacterium bovis.

A partir del resultado del cultivo, la ausencia de compromiso sistémico de otra enfermedad y tras descartar una posible alteración del sistema inmunitario, se arribó al diagnóstico de osteomielitis secundaria a la vacuna BCG.

El tratamiento indicado por el Servicio de Infectología consistió en fármacos antituberculosos durante 10 meses, según el siguiente esquema:

- Dos meses, por vía endovenosa: rifampicina + isoniacida + etambutol + estreptomicina (inicialmente en el Hospital y, luego, con internación domiciliaria)

- Ocho meses, por vía oral: rifampicina + isoniacida

La evolución de la paciente fue muy favorable, sin dolor, ni secuelas motoras, ni daño esquelético residual, sin compromiso de la fisis, ni del crecimiento del miembro.

Se puede observar la evolución de la lesión ósea en los controles radiográficos posoperatorios (Figuras 3-5).

\section{DISCUSIÓN}

\section{Vacuna BCG}

La vacuna BCG es una preparación liofilizada, constituida por micobacterias vivas obtenidas de un cultivo de bacilos bovinos atenuados. Todas las cepas de la vacuna actuales derivan del aislado original de M. bovis que Calmette y Guérin sometieron a numerosos ciclos de atenuación durante un período de 13 años (1909-1921). Estas cepas tienen disminuida la virulencia, pero conservan la capacidad de proteger contra la tuberculosis, crear sensibilidad a la tuberculina y dejar cicatriz en la mayoría de los vacunados. 

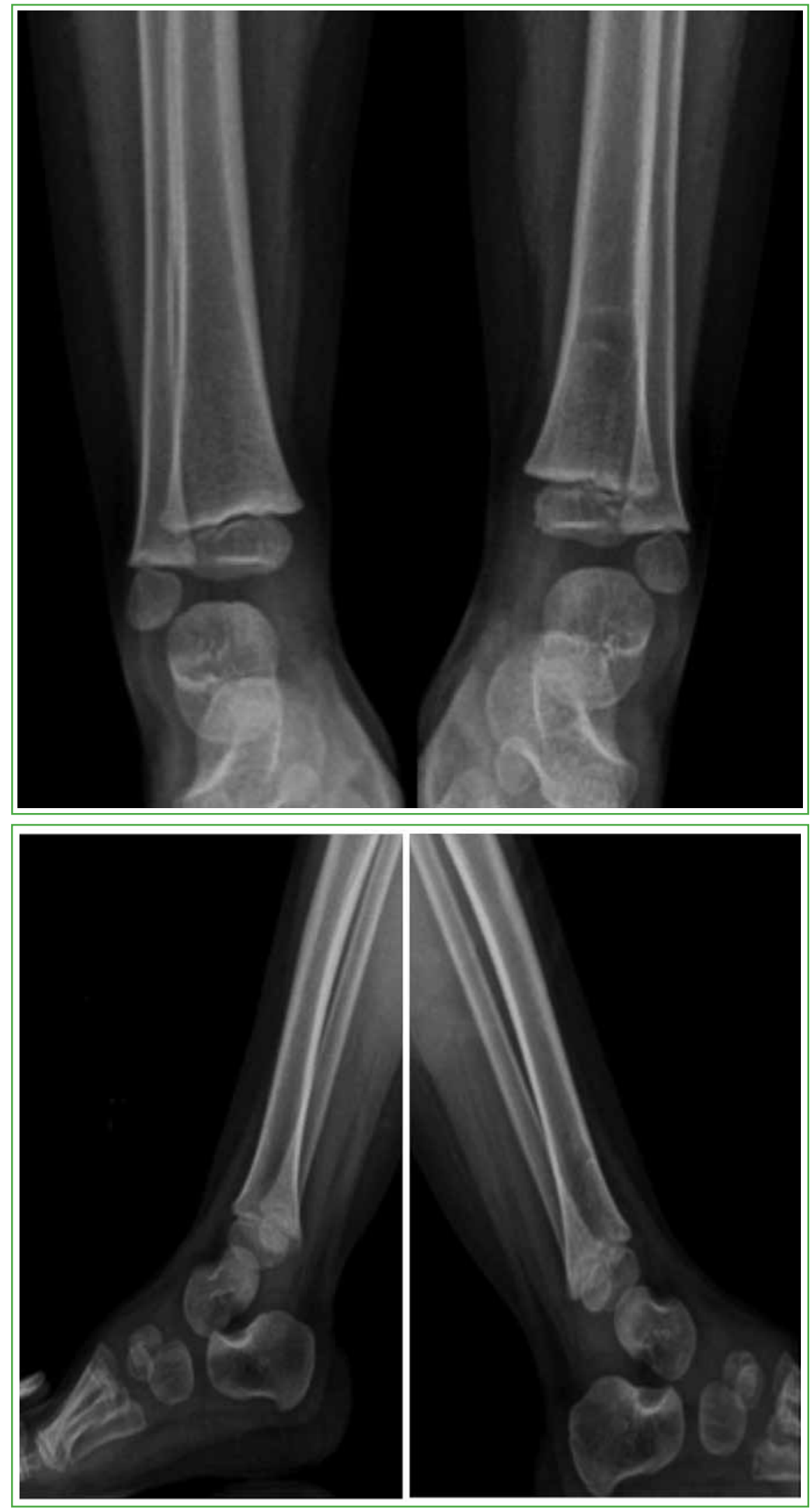

Figura 3. Radiografías de frente y de perfil, de ambos tobillos, al año de la intervención. 

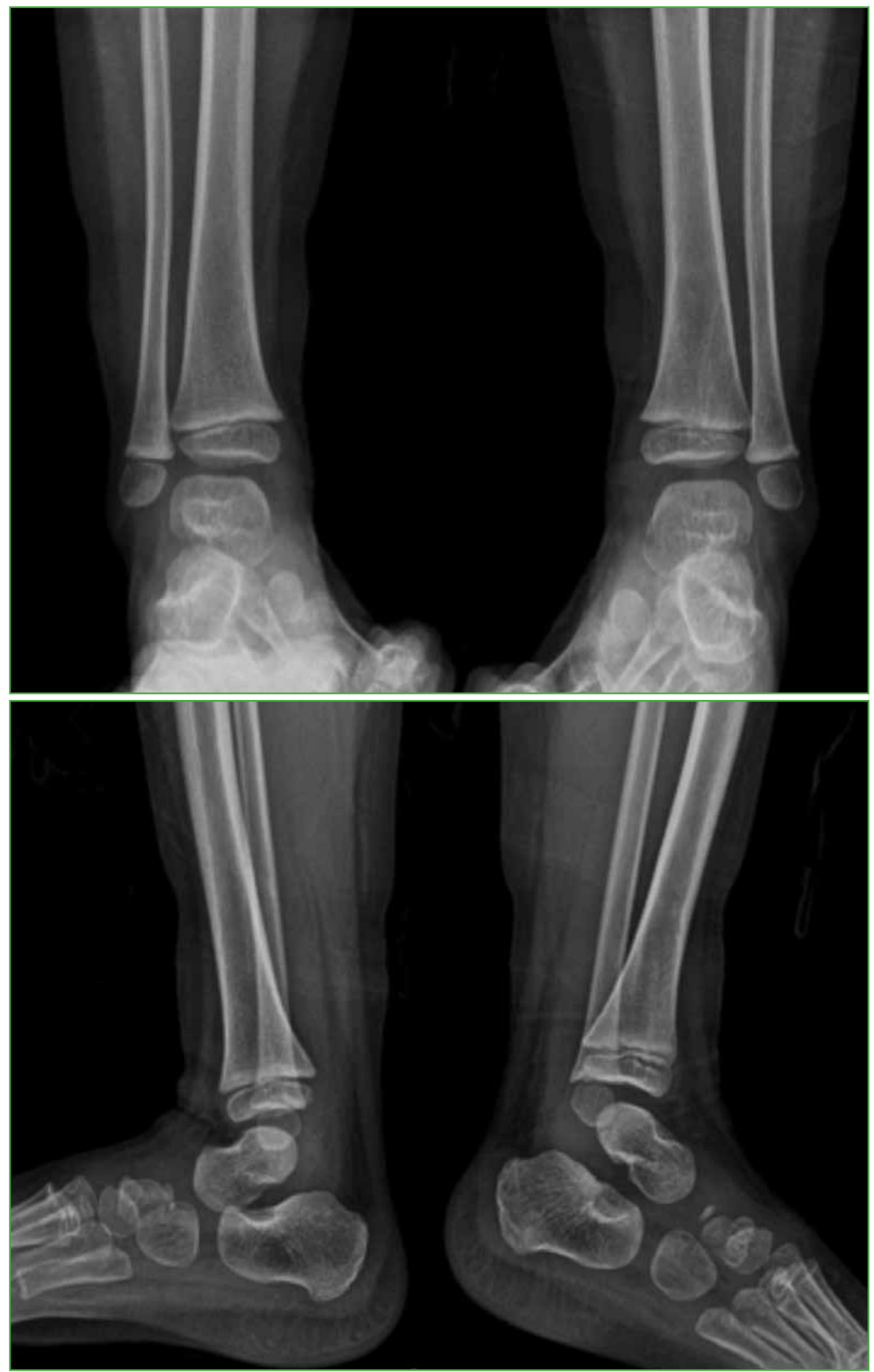

Figura 4. Radiografías de frente y de perfil, de ambos tobillos, a los dos años de la intervención. 

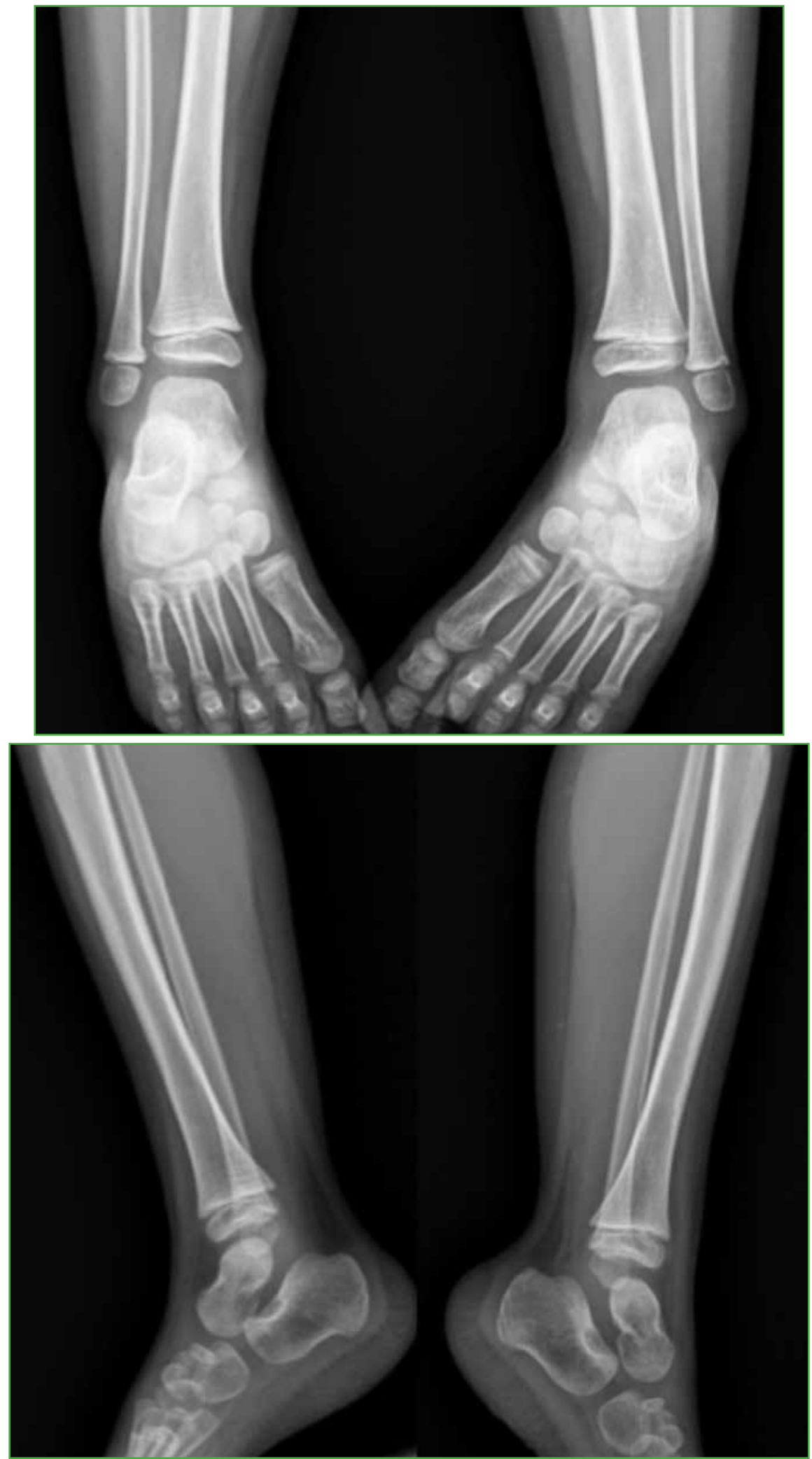

Figura 5. Radiografías de frente y de perfil, de ambos tobillos, a los 2 años y 6 meses de la intervención 
Con el transcurso de los años, el paso por diferentes medios de cultivo y distintas condiciones, según cada laboratorio, se obtuvieron diversas subcepas de BCG con diferencias fenotípicas y genotípicas. Estas toman el nombre del laboratorio o ciudad donde han sido cultivadas. La cantidad de unidades formadoras de colonias por mililitro (UFC/ml) varía en cada una de ellas. Ejemplos:

- Danesa: de 2 a 8 millones UFC/ml

- Glaxo: de 2 a 10 millones UFC/ml

- Moreau: de 2 a 8 millones UFC/ml

- Pasteur: de 2 a 8 millones UFC/ml

- Tokio: 30 millones UFC/ml

- Laboratorio Central de Salud Pública: Cepa Pasteur (Provincia de Buenos Aires): de 3 a 8 millones UFC/ml

La potencia de la vacuna depende de la cepa utilizada, la dosis, la correcta conservación, el manejo y la aplicación. Se requiere un sistema de refrigeración y vigilancia permanente de la temperatura y el transporte hasta su uso. No debe exponerse a la acción de la luz solar ni a otra fuente de rayos ultravioleta. Actualmente, el método de preparación está estandarizado, la Organización Mundial de la Salud es el organismo responsable de controlar la calidad de la vacuna.

El objetivo de la vacuna es limitar la multiplicación de bacilos tuberculosos y su diseminación hematógena tras la infección primaria. Ejerce su mayor efecto protector sobre las formas graves diseminadas, miliar o meníngea. No actúa sobre la reinfección exógena y no está comprobado su papel en la reactivación endógena.

La vacuna BCG es la más difundida en el mundo y, en la Argentina, está incorporada en el Programa Ampliado de Inmunización desde 1974. En nuestro país, la vacunación inicial se efectúa en todo recién nacido con un peso al nacer de $2 \mathrm{~kg}$ o más antes de salir de la maternidad. Los niños de 1 mes de vida a 6 años que no hubieran recibido la vacuna BCG al nacer (sin cicatriz o registro de vacunación), deben ser vacunados una vez descartada la tuberculosis, siempre que no sufran alteraciones inmunológicas.

Se estima que la inmunidad conferida por la vacuna no es mayor que 10 años.

El riesgo de reacciones adversas está asociado a la cepa utilizada por el fabricante, el exceso en la dosis, la edad del niño, la técnica de aplicación y, en algunos casos, a alteraciones de la inmunidad. Las reacciones pueden ser:

\section{Locales:}

- Ulceración prolongada

- Formación de abscesos

- Adenopatías locales de gran tamaño o supuradas, con fistulización o sin ella (puede considerarse normal la presencia de una pequeña adenopatía axilar)

- Reacción lupoide (símil lupus)

Se trata de reacciones leves o moderadas que curan espontáneamente, aunque la evolución sea prolongada. Ninguna requiere tratamiento medicamentoso local, general o quirúrgico.

\section{Generales:}

- Infección diseminada por vacuna BCG (de 1 a 12 meses posvacunación): 2 /1.000.000 de dosis aplicadas.

- Osteítis u osteomielitis por vacuna BCG (de 1 a 12 meses posvacunación): 1-700/1.000.000 de dosis aplicadas.

Estas complicaciones se han producido, sobre todo en pacientes con síndrome de la inmunodeficiencia adquirida u otra enfermedad que altere el sistema inmunológico, que han recibido la vacuna por error. ${ }^{4}$

La vacuna BCG está contraindicada en pacientes con:

- Enfermedades con grave compromiso del estado general

- Afecciones generalizadas de la piel

- Enfermedades infecciosas (especialmente sarampión y varicela), esperar un mes de transcurridas estas infecciones.

- Inmunodepresión congénita o adquirida

- Tratamiento prolongado con corticoides o drogas inmunodepresoras

- Recién nacidos prematuros que pesan $<2 \mathrm{~kg}$. Una vez superado dicho peso debe aplicarse la vacuna. ${ }^{2}$ 
La osteomielitis u osteítis secundaria a la vacuna BCG es una complicación poco común, aunque grave, que se ha notificado principalmente en la Península Escandinava y en Europa del Este, y que, por lo general, se asocia a cambios en la cepa de la vacuna. Se notificó un incremento en el número de casos de osteítis a 35 por millón en Checoslovaquia después de cambiar de la cepa de Praga a la rusa (Lotte y cols., 1988). ${ }^{5}$ Finlandia y Suecia notificaron aumentos de osteítis después de 1971, cuando cambiaron a una cepa de Gotemburgo producida en Dinamarca. Suecia reportó tasas muy altas de hasta 1 en 3000 personas vacunadas, las cuales disminuyeron rápidamente cuando el programa nacional cambió a la vacuna de la cepa danesa (Copenhague, 1331). Más recientemente, los reportes de osteítis han sido menos frecuentes. ${ }^{3,6}$ En 2015, Lin y cols. llevaron a cabo una revisión sistemática sobre el manejo de la osteomielitis/osteítis secundaria a la vacuna BCG en niños inmunocompetentes. Analizaron 34 estudios publicados entre 1976 y 2013, donde se estudiaron 331 casos de osteomielitis secundaria a la vacuna BCG. Los resultados mostraron que, en su gran mayoría, el compromiso óseo está dado por una lesión osteolítica bien definida y única. El 55,6\% de las lesiones óseas estaban ubicadas en los miembros inferiores, mientras que el 15,4\% afectaba los miembros superiores. En el 26\%, comprometían el esqueleto axial (columna, costillas, esternón, etc.) y, solo en el 3\%, la afección ósea era en más de un hueso en simultáneo. En el 97,6\% de los casos, la evolución y resolución clínicas fueron favorables, sin secuelas. Los autores llegaron a la conclusión de que, en pacientes pediátricos inmunocompetentes, el tratamiento farmacológico antituberculoso sería suficiente y la mejor opción terapéutica. Los procedimientos quirúrgicos son útiles, sobre todo para el diagnóstico de la infección, por ejemplo, la biopsia ósea por aspiración. Se deberían evitar cirugías más agresivas, como el curetaje de la lesión, en niños cuando el esqueleto aún está inmaduro, ya que pueden asociarse a alteraciones esqueléticas permanentes secundarias a la lesión de la fisis (desejes o discrepancia de longitud de los miembros). ${ }^{7}$

Nuestra experiencia coincide con la revisión bibliográfica de Lin y cols., ya que nuestra paciente presentó una única lesión osteolítica en los miembros inferiores. Al mismo tiempo, el tratamiento farmacológico y sintomático fue suficiente para lograr buenos resultados funcionales, sin evidencia de secuelas de la enfermedad, ni clínicas, ni radiológicas.

Consideramos que es importante sospechar esta rara etiología en lactantes que presenten una lesión ósea lítica, sin signos claros de infección bacteriana y ni enfermedad neoplásica. Ante dicho cuadro, en el estudio bacteriológico, se debe solicitar el análisis de gérmenes atípicos, como hongos, bacterias acidorresistentes y micobacterias, en las muestras obtenidas en la biopsia. Como es un cuadro infrecuente y la experiencia en su manejo es escasa, nos vemos limitados para poder establecer pautas estandarizadas de un tratamiento de elección.

Conflicto de intereses: Los autores no declaran conflictos de intereses.

ORCID ID de E. J. Levy: https://orcid.org/0000-0002-9158-8617

\section{BIBLIOGRAFÍA}

1. World Health Organization. Global Tuberculosis Report 2015. Geneva: World Health Organization; 2015. https://apps.who.int/medicinedocs/documents/s22199en/s22199en.pdf

2. Ministerio de Salud de la República Argentina. Recomendaciones Nacionales de Vacunación Argentina 2012. http://www.msal.gob.ar/images/stories/bes/graficos/0000000451cnt-2013-06_recomendaciones-vacunacion-argentina-2012.pdf

3. Organización Mundial de la Salud. Hoja de información sobre las tasas observadas de reacciones a vacunas. Vacuna Bacilo de Calmette-Guérin (BCG); 2012.

https://www.who.int/vaccine_safety/initiative/tools/BCG_Vaccine_rates_information_sheet_ES.pdf?ua=1

4. Ministerio de Salud de la República Argentina. Vacunación Segura: Vigilancia de eventos supuestamente atribuibles a la vacunación o inmunización (ESAVI); 2012.

http://www.msal.gob.ar/images/stories/bes/graficos/0000000448cnt-2014-01_manual-vacunacion-segura-esavi.pdf 
5. Lotte A, Wasz-Hockert O, Poisson N, Engbaek H, Landmann H, Quast U, et al. Second IUATLD study on complications induced by intradermal BCG-vaccination. Bull Int Union Tuberc Lung Dis 1988;63(2):47-59. PMID: 3066422

6. Organización Mundial de la Salud. Documento de posición de la OMS sobre las vacunas BCG. Boletín Epidemiológico Semanal 2018;8(93):73-96.

http://www9.who.int/immunization/policy/position_papers/pp_bgc_2018_ES.pdf

7. Lin WL, Chiu NC, Lee PH, Huang AS, Huang FY, Chi H, et al. Review management of Bacillus Calmette-Guérin osteomyelitis/osteitis in immunocompetent children-A systematic review. Vaccine 2015;33(36):4391-7. https://doi.org/10.1016/j.vaccine.2015.07.039 